\title{
Chironomid Fauna in the Emergent Plant Zone of Lake Kasumigaura, Japan
}

\author{
Ryuhei UENo, Toshio IWAKUMa \\ and Seiichi NOHARA
}

\begin{abstract}
The chironomid fauna was studied in the emergent plant zone of Lake Kasumigaura in May, August and November of 1986. A total of 2,181 adults were reared and emerged from plant samples and sediments in the emergent plant zone. We identified 1957 specimens belonging to 31 species of 18 genera. Ten species were abundant, of which five were recovered from plant samples, two from sediments and others from both. Species composition did not differ much from May to August, which might have corresponded with the change of in situ larval density. The abundance of the chironomids was highest in May and low in August for plant samples, while it was highest in August for sediments. On plant samples, it correlated with the chlorphyll a amount on the reed stems, which was higher in May than in August. In November, only two species predominated. Species composition differed more markedly among substratum types than among localities in the lake. Some chironomid species seemed to select specific sediments. Chironomid fauna in littoral hydrophyte zones was more diverse than in pelagic zones in Lake Kasumigaura.
\end{abstract}

Key words : Chironomidae, Lake Kasumigaura, littoral zone, emergent plants

\section{Introduction}

Chironomid larvae live in almost all types of water bodies. They often dominate in eutrophic lakes or polluted rivers. Sometimes they prosper in the shallow littoral hydrophyte zones (Boerger et al., 1982). In Japan, chironomid fauna has been studied in some eutrophic lakes or ponds (e.g. SASA, 1985a, b; SASA and KawAI, 1987). However, the chironomid fauna in littoral zones of lakes or ponds, especially hydrophyte zones, has not been studied sufficiently (Kondo and Hamashima, 1985; Kondo, 1988), though the important role of the littoral zone of lakes has been pointed out by some authors (e.g. Wetzel and CoRners, 1979). As for Lake Kasumigaura, ecological studies on chironomids in the pelagic zone were made by Iwakuma et al. (1984) and Iwakuma (1987). Several authors reported on the benthic fauna in the sublittoral zone (e.g. MIYADI, 1932; TsudA and Morishita, 1973), and some chironomid species collected around the lake were described by SASA (1979). Most of these studies focused on pelagic zones. Recently, primary production of algae was studied minutely in the hydrophyte zone of Lake Kasumigaura ( $\mathrm{TA}$. KAMURA et al., 1990), though benthic fauna have yet to be studied in the hydrophyte zone.

Studies on the habitat preference of the chironomids between emergent plant and sediment have been very few (LeARner et al., 1989; MASON and BRYANT, 1975), whereas interactions between hydrophytes and chironomid larvae have been drawing attention for a long time, i.e. direct feeding on plants (McGaHA, 1952; NODA et al., 1986), feeding on the epiphyton (MenzIE, 1981) or utilization of the decomposed plants (Smock and Stoneburner, 1980). Most of these studies have been conducted on floating and submersed plants in lotic systems.

In the present paper, we studied the epiphytic 


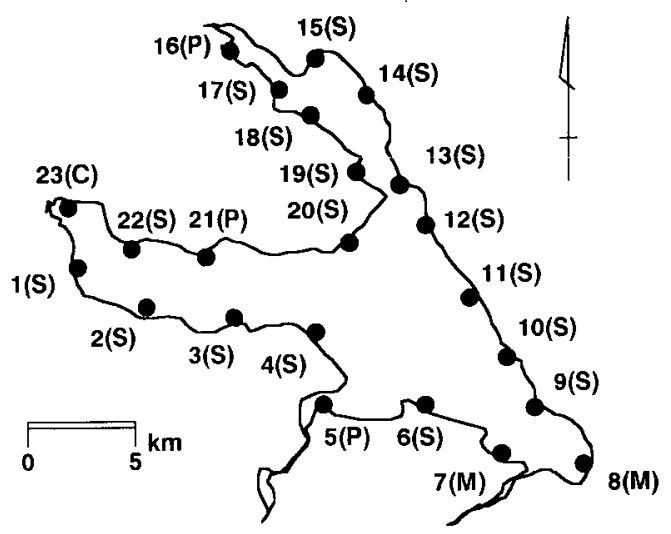

Fig. 1. Map of sampling stations with major sediment type.

$\mathrm{S}$ : sand, $\mathrm{M}$ : mud, $\mathrm{C}$ : cobble, $\mathrm{P}$ : plant debris.

and benthic chironomid fauna in emergent plant zones in Lake Kasumigaura and the habitat specificity of some chironomid species.

\section{Study sites}

Lake Kasumigaura is a hypertrophic lake with a surface area of $171 \mathrm{~km}^{2}$, a perimeter of $136 \mathrm{~km}$ and mean depth of about $4 \mathrm{~m}$. Since the lake is a shallow and has a complicated shoreline, hydrophytes grow thick in the littoral zone. Total vegetation areas of emergent, floating and submerged plants were estimated as $3.0 \mathrm{~km}^{2}, 0.8 \mathrm{~km}^{2}$ and $3.6 \mathrm{~km}^{2}$, respectively (SAKURAI, 1981). We selected 23 sampling stations in emergent plant zones at intervals of $4 \mathrm{~km}$ to $5 \mathrm{~km}$ along the shoreline (Fig. 1). Lake Kasumigaura consists of three large bays, i.e. Tsuchiurairi Bay (Stas. 1-3, 21-23), Takahamairi Bay (Stas. 13-19), Edosakiiri Bay (Sta. 5), and a main basin (other stations). Total phosphorus concentration was generally higher in Takahamairi Bay and Tsuchiurairi Bay than in the Main Basin and Edosakiiri Bay in 1986 (AIZAKI et al., 1988a; AIZAKI et al., 1988b). Surface sediment type was sand or mud at most stations, but cobble predominated in Sta. 23, and plant debris in Stas. 5, 16 and 21. Table 1 shows some botanical parameters at each station. Dominant hydrophyte species was a reed, Phragmites australis (Cav.) Trin. ex Steud., except at Sta. 6 and Sta. 9. A cattail, Typha angustifolia $\mathrm{L}$. dominated at Sta. 6, and no hydrophytes were found at Sta. 9. Though hydrophytes were dead by November, dead stems of $P$. australis remained as substrata for epiphyton; woolly masses of epiphytic organisms adhered to their surface throughout the winter.

\section{Materials and methods}

In 1986, emergent plants and sediments were collected on 21 May (Stas. 1-21), 6 June (Stas. 22, 23), 27 August (Stas. 1-16), 29 August (Stas. 17-23), 12 November (Stas. 1-13) and 13 November (Stas. 14-23) 1986. Emergent plants were cut off at the bottom of their stems from a $33 \times$ $33 \mathrm{~cm}$ quadrat. Submerged parts of emergent plants were then cut off and stored in polyethylene bags. Mean density and mean surface area of emergent plants at 22 stations (except Sta. 9) were estimated in May and August. Mean density of emergent plants was $134 \mathrm{~m}^{-2}$ and $144 \mathrm{~m}^{-2}$ in May and August, respectively. Mean surface area of emergent plants, multiplying mean perimeter of stems by water depth and mean density, was $0.93 \mathrm{~m}^{2} \cdot \mathrm{m}^{-2}$ and $1.03 \mathrm{~m}^{2}$ - $\mathrm{m}^{-2}$ in May and August, respectively. Sediments were collected from about $0.1 \mathrm{~m}^{2}$ area of bottom surface within emergent plant stands with a $D$-frame net $(25 \times 20 \mathrm{~cm}, 0.3 \mathrm{~mm}$ opening). Duplicate emergent plant samples and a sediment sample were taken at each station and transported to the laboratory. One of the plant samples and the sediment sample from each station were transferred to transparent plastic containers $30 \mathrm{~cm}$ in diameter and $12 \mathrm{~cm}$ in height). The containers were filled with tap water to a depth of about $7 \mathrm{~cm}$, and air was supplied with a diaphragm pump. Each container was covered with fine nylon net fixed with a rubber band and was kept in doors under $12 \mathrm{hr}$ light and $12 \mathrm{hr}$ dark photoperiod at $20^{\circ} \mathrm{C}$ (May and August) or $15^{\circ} \mathrm{C}$ (November). Chironomid midges, which emerged from samples in the containers were collected with an aspirator every two or three days for about two months after rearing started. Collected midges were anesthetized with diethyl ether and were preser- 
Table 1. Some sedimentary and botanical parameters at each station.

\begin{tabular}{|c|c|c|c|c|c|c|c|c|c|c|c|}
\hline \multirow[b]{2}{*}{ Stat } & \multirow[b]{2}{*}{$\begin{array}{c}\text { Loss on ignition }{ }^{a} \\
\text { of surface } \\
\text { sediment } \\
(\%) \\
(n=3) \\
\end{array}$} & \multicolumn{4}{|c|}{ May } & \multicolumn{4}{|c|}{ August } & \multicolumn{2}{|c|}{ November ${ }^{b}$} \\
\hline & & $\begin{array}{l}\text { Depth } \\
(\mathrm{cm})\end{array}$ & $\begin{array}{l}\text { Density } \\
\text { of } \\
\text { plants } \\
\left(\mathrm{m}^{-2}\right) \\
(n=4)\end{array}$ & $\begin{array}{l}\text { Surface } \\
\text { area of } \\
\text { plants } \\
\left(\mathrm{m}^{2} \cdot \mathrm{m}^{-2}\right) \\
(n=1)\end{array}$ & 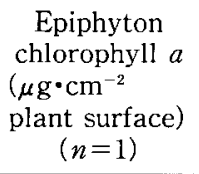 & $\begin{array}{l}\text { Depth } \\
(\mathrm{cm})\end{array}$ & $\begin{array}{l}\text { Density } \\
\text { of plants } \\
\left(\mathrm{m}^{-2}\right) \\
(n=3)\end{array}$ & $\begin{array}{l}\text { Surface } \\
\text { Area of } \\
\text { plants } \\
\left(\mathrm{m}^{2} \cdot \mathrm{m}^{-2}\right) \\
(n=1)\end{array}$ & $\begin{array}{l}\text { Epiphyton } \\
\text { chlorophyll } a \\
\left(\mu \mathrm{g} \cdot \mathrm{cm}^{-2}\right. \\
\text { plant surface }) \\
\quad(n=1)\end{array}$ & $\begin{array}{l}\text { Depth } \\
(\mathrm{cm})\end{array}$ & $\begin{array}{l}\begin{array}{l}\text { Epiphyton } \\
\text { chlorophyll } a \\
\left(\mu \mathrm{g} \cdot \mathrm{cm}^{-2}\right. \\
\text { plant surface }) \\
\quad(n=5)\end{array}\end{array}$ \\
\hline 1 & $2.0 \pm 0.6^{c}$ & 31 & $77 \pm 13^{c}$ & 0.63 & 2.0 & 26 & $131 \pm 98^{c}$ & 0.98 & 0.7 & 36 & $16.0 \pm 7.9^{c}$ \\
\hline 2 & $2.1 \pm 0.3$ & 39 & $139 \pm 27$ & 1.46 & 2.3 & 39 & $189 \pm 53$ & 2.04 & 0.4 & 57 & $7.3 \pm 5.4$ \\
\hline 3 & $0.7 \pm 0.0$ & 38 & $83 \pm 17$ & 0.79 & 3.2 & 50 & $71 \pm 30$ & 0.63 & 5.8 & 25 & $14.4 \pm 4.7$ \\
\hline 4 & $1.6 \pm 0.3$ & 18 & $118 \pm 5$ & 0.53 & 9.5 & 25 & $173 \pm 42$ & 0.66 & 1.5 & 50 & $14.6 \pm 7.4$ \\
\hline 5 & $1.8 \pm 1.0$ & 38 & $117 \pm 34$ & 1.03 & 0.6 & 23 & $44 \pm 26$ & 0.48 & 0.7 & 40 & $0.9 \pm 0.6$ \\
\hline 6 & $0.9 \pm 0.2$ & 17 & $130 \pm 27$ & 0.42 & 1.0 & 18 & $144 \pm 03$ & 0.50 & 2.2 & 18 & $8.2 \pm 4.2$ \\
\hline 7 & $0.8 \pm 0.1$ & 30 & $169 \pm 78$ & 1.01 & 2.4 & 26 & $118 \pm 09$ & 0.62 & 0.9 & 55 & $14.2 \pm 16.8$ \\
\hline 8 & $2.7 \pm 0.7$ & 40 & $60 \pm 8$ & 0.62 & 1.8 & 38 & $96 \pm 63$ & 0.95 & 0.8 & 30 & $11.4 \pm 9.0$ \\
\hline 9 & $0.6 \pm 0.1$ & - & 0 & 0 & - & - & 0 & 0 & - & 85 & - \\
\hline 10 & $1.4 \pm 0.3$ & 45 & $74 \pm 8$ & 0.82 & 6.4 & 38 & $117 \pm 15$ & 2.07 & 0.5 & 52 & $13.9 \pm 6.3$ \\
\hline 11 & $1.0 \pm 0.3$ & 22 & $150 \pm 33$ & 0.71 & 2.7 & 45 & $227 \pm 73$ & 1.21 & 0.6 & 25 & $11.8 \pm 3.4$ \\
\hline 12 & $1.1 \pm 0.3$ & 24 & $89 \pm 1$ & 0.56 & 6.4 & 32 & $153 \pm 19$ & 1.00 & 0.2 & $4 \dot{5}$ & $8.6 \pm 1.7$ \\
\hline 13 & $1.3 \pm 0.8$ & 20 & $156 \pm 50$ & 0.72 & 4.1 & 34 & $165 \pm 47$ & 0.77 & 3.3 & 38 & $21.5 \pm 3.6$ \\
\hline 14 & $0.6 \pm 0.1$ & 23 & $139 \pm 24$ & 0.84 & 6.2 & 36 & $167 \pm 26$ & 0.84 & 3.4 & 28 & $1.6 \pm 0.8$ \\
\hline 15 & $1.8 \pm 1.1$ & 38 & $164 \pm 34$ & 1.46 & 1.3 & 49 & $183 \pm 49$ & 1.53 & 0.5 & 47 & $1.7 \pm 0.4$ \\
\hline 16 & $59.9 \pm 6.5$ & 5 & $61 \pm 10$ & 0.08 & 4.3 & 5 & $82 \pm 23$ & 0.10 & 1.3 & 6 & $1.2 \pm 0.6$ \\
\hline 17 & $0.6 \pm 0.1$ & 39 & $111 \pm 23$ & 0.92 & 6.2 & 55 & $152 \pm 15$ & 1.11 & 1.1 & 49 & $6.3 \pm 8.5$ \\
\hline 18 & $1.0 \pm 0.2$ & 42 & $136 \pm 24$ & 1.48 & 6.8 & 36 & $139 \pm 26$ & 1.48 & 1.3 & 45 & $2.8 \pm 0.4$ \\
\hline 19 & $0.8 \pm 0.4$ & 43 & $100 \pm 7$ & 1.23 & 5.1 & 42 & $150 \pm 29$ & 1.74 & 0.2 & 47 & $11.3 \pm 11.5$ \\
\hline 20 & $0.9 \pm 0.3$ & 25 & $130 \pm 8$ & 0.73 & 8.2 & 41 & $137 \pm 30$ & 0.75 & 4.8 & 46 & $5.5 \pm 3.1$ \\
\hline 21 & $1.5 \pm 0.4$ & 20 & $207 \pm 14$ & 1.08 & 11.2 & 38 & $251 \pm 51$ & 1.28 & 3.0 & 47 & $14.7 \pm 12.1$ \\
\hline 22 & $2.0 \pm 0.4$ & 25 & $243 \pm 47$ & 1.55 & 5.2 & 29 & $134 \pm 25$ & 0.88 & 0.8 & 35 & $6.6 \pm 4.1$ \\
\hline 23 & $1.3 \pm 0.4$ & 27 & $287 \pm 37$ & 1.73 & 4.7 & 28 & $153 \pm 33$ & 1.08 & 0.9 & 37 & $11.4 \pm 7.8$ \\
\hline
\end{tabular}

${ }^{a}$ Loss on ignition determined in November.

${ }^{b}$ In November, density and surface area of reeds was not determined.

$c$ Mean value \pm standard deviation $\left(\sigma_{n-1}\right)$. 
ved in $70 \%$ ethanol together with pupal exuviae. Some specimens were dissected under binoculars and mounted on slide glasses for identification (SASA, 1978; SASA and HASEGAWA, 1983). They were identified according to the key by Pinder (1978), SASA (1989) and Cranston and Oliver (1988) and counted. Each sample was calculated for the following Simpson's index of concentration (C) (Simpson, 1949):

$$
C=\sum_{i}\left(n_{i}\right)^{2} / N^{2}
$$

where $n_{i}$ is the number of individuals of the species $i$ and $N$ is the total number of individuals emerged.

Chlorophyll $a$ amount of epiphyton in each plant sample was determined as follows: Dead stems of hydrophytes and epiphytic matter removed from live stems in some of the other plant samples were put together into a screw -capped bottle in May and August, or put individually into screw-capped glass tubes in November. They were extracted with $100 \%$ methanol and stored in the dark for a week. The solution was poured into a $10 \mathrm{ml}$ glass tube, centrifuged at 3,000 r.p.m., and the optical density of the supernatant was measured at 750,664 $\mathrm{nm}$ in wavelength with a spectrophotometer (Beckman DU-30). Chlorophyll $a$ amount was calculated based on MARKER et al. (1980), and plant surface areas per unit of lake bottom area were estimated.

Three core samples were taken to determine loss on ignition of sediment at each station in November. Surface sediment to a depth of $5 \mathrm{~cm}$ was extruded from each core. Then the three sediments from each station were homogenized and mixed. A small amount of the mixture up to $150 \mathrm{mg}$ was transferred into a ceramic pot, oven-dried at $120^{\circ} \mathrm{C}$ for $1 \mathrm{hr}$ and combusted at $500^{\circ} \mathrm{C}$ in an electric oven for $8 \mathrm{hr}$. Loss on ignition was calculated from the difference between weights at $120^{\circ} \mathrm{C}$ and at $500^{\circ} \mathrm{C}$.

\section{Results}

Loss on ignition varied from $0.6 \%$ to $2.7 \%$, except at Sta. 16 where organic matter accounted for $59.9 \%$. Mean epiphyton chlorophyll $a$ of
22 stations was $4.6 \mu \mathrm{g} \cdot \mathrm{cm}^{-2}$ plant surface in May, decreasing to $1.6 \mu \mathrm{g} \cdot \mathrm{cm}^{-2}$ plant surface in August and reaching a maximum $9.4 \mu \mathrm{g} \cdot \mathrm{cm}^{-2}$ plant surface in November (Table 1).

During this study, 2,181 chironomid midges were obtained, and 1957 specimens belonging to 31 species were identified (Table 2). A total of 1,529 specimens of 24 species and 428 specimens of 22 species emerged from hydrophytes and sediments, respectively. Of these species, one was a member of Tanypodinae, one of Diamesinae, nine were of Orthocladiinae and 20 of Chironominae. Still unidentified were 224 specimens, 207 of which were females of the tribe Tanytarsini. Many of them was identical to Paratanytarsus grimmii (SchneIDer, 1885) $(=P$. parthenogeneticus (FreEman, 1961)) in SaSA (1979), which is known to breed usually by parthenogenesis. Table 3 shows number of species $(S)$, number of identified individuals $(N)$ and concentration indices $(C)$. Both $S$ and $N$ were high in May and low in November. The $C$ values were frequent at $0.3-0.4$ in May or August and at 1.0 in November for plant samples. Though the $C$ values showed no noticeable differences among three occasions on sediment samples, median value was low in May $(0$. $5)$ and high in August (0.9) and November (1.0). So the chironomid fauna was more diverse in May or August than in November regardless of substratum.

A combination of ten leading species accounted for $84.5 \%$ of the chironomid midges emerged: Cricotopus sylvestris, Hydrobaenus pilipes, Limnophyes tamakitanaides, Chironomus yoshimatsui, Dicrotendipes nervosus, Dicrotendipes pelochloris, Glyptotendipes tokunagai, Polypedilum cultellatum, Polypedilum nubifer and Tanytarsus oyamai.

Five species, C. sylvestris, L. tamakitanaides, $D$. nervosus, D. pelochloris and P. cultellatum, emerged in larger numbers from hydrophytes than from sediments. Two species, $P$. nubifer and T. oyamai, emerged in larger numbers from sediments than hydrophytes. Three other species, $C$. yoshimatsui, $H$. pilipes and $G$. tokunagai, emerged well from both types of substratum. C. sylvestris, D. pelochloris, $G$. to- 
kunagai and $P$. cultellatum emerged mainly from May samples. C. yoshimatsui, $P$. nubifer and $T$. oyamai emerged mainly from August samples. $H$. pilipes emerged only from November samples. All specimens of $L$. tamakitanaides but one emerged from November samples. D. nervosus emerged from both May and August samples.

Cryptochironomus sp. emerged only from sediments in every season, though it was not a major species.

Four species of Cricotopus were recovered in this study. Cricotopus sylvestris emerged mainly from hydrophytes in May and exclusively from

Table 2. Numbers of individuals emerging for each species by substratum and season

\begin{tabular}{|c|c|c|c|c|c|}
\hline \multirow[t]{2}{*}{ Species } & \multicolumn{2}{|c|}{ Substrata } & \multicolumn{3}{|c|}{ Season } \\
\hline & Plants & Sediments & May & August & November \\
\hline \multicolumn{6}{|l|}{ Tanypodinae } \\
\hline Ablabesmyia monilis (LiNNAEUS) & & 2 & & 2 & \\
\hline \multicolumn{6}{|l|}{ Diamesinae } \\
\hline Potthastia gaedii (MEIGEN) & 1 & & & & \\
\hline \multicolumn{6}{|l|}{ Orthocladiinae } \\
\hline Cricotopus bicinctus (MEIGEN) & 18 & 4 & 2 & 1 & 19 \\
\hline Cricotopus bimaculatus TокиNAGA & 4 & & & & 4 \\
\hline Cricotopus sylvestris (FABRICIUS) & 542 & 16 & 459 & 95 & 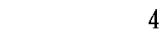 \\
\hline Cricotopus tricinctus (MeIGEN) & 1 & 1 & 2 & & \\
\hline Hydrobaenus pilipes (МАLLOCH) & 199 & 46 & & & 245 \\
\hline Limnophyes immucronatus $\mathrm{S}_{\text {ATHER }}$ & 1 & & & & 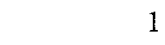 \\
\hline Limnophyes tamakitanaides $\mathrm{S}_{\mathrm{ASA}}$ & 44 & & & 1 & 43 \\
\hline Orthocladius glabripennis (Goеtghebuer) & 1 & & & & 1 \\
\hline Paratrichocladius mufiventris (MEIGEN) & 3 & & 1 & & 2 \\
\hline \multicolumn{6}{|l|}{ Chironominae: Chironomini } \\
\hline Chironomus circumdatus (KIEFFER) & & 1 & & 1 & \\
\hline Chironomus kiiensis TокUNAGA & 3 & 8 & 2 & 9 & \\
\hline Chironomus samoensis EDWARDS & & 2 & & 2 & \\
\hline Chironomus yoshimatsui Martin et Sublette & 31 & 49 & 6 & 74 & \\
\hline Cladopelma viridula (LinNaEus) & 1 & 1 & 2 & & \\
\hline Cryptochironomus sp. nr. fulvus (JoHANNSEN) & & 21 & 6 & 10 & .5 \\
\hline Dicrotendipes nervosus (STAEGER) & 54 & 5 & 24 & 35 & \\
\hline Dicrotendipes pelochloris (KIEFFER) & 344 & 24 & 307 & 61 & \\
\hline Glyptotendipes fujisecundus (SASA) & 1 & & 1 & & \\
\hline Glyptotendipes tokunagai $\mathrm{S}_{\mathrm{ASA}}$ & 80 & 20 & 80 & 20 & \\
\hline Pentapedilum shirokanense $\mathrm{S}_{\mathrm{ASA}}$ & 5 & 4 & 1 & 8 & \\
\hline Polypedilum cultellatum GoETGHEBuER & 159 & 2 & 114 & 44 & 3 \\
\hline Polypedilum japonicum (TокиNAGA) & & 2 & 2 & & \\
\hline Polypedilum nubeculosum (MEIGEN) & & 3 & & 3 & \\
\hline Polypedilum nubifer (Skuse) & 29 & 130 & & 157 & 2 \\
\hline Stenochironomus membranifer Ү Үмамото & 1 & & & 1 & \\
\hline \multicolumn{6}{|l|}{ Chironominae: Tanytarsini } \\
\hline Cladotanytarsus vandernoulpi (EDWARDS) & 1 & 10 & 7 & 4 & \\
\hline Rheotanytarsus aestuarius (ТокUNAGA) & 2 & & 1 & 1 & \\
\hline Tanytarsus oyamai SASA & & 69 & 1 & 68 & \\
\hline Tanytarsus unagiseptimus SASA & $\dot{4}$ & 8 & 6 & 6 & \\
\hline Unidentified & 156 & 68 & 162 & 54 & 8 \\
\hline Total & 1,685 & 496 & 1,186 & 657 & 338 \\
\hline Number of species & 24 & 22 & 19 & 21 & 12 \\
\hline
\end{tabular}


Table 3. Number of species $(S)$, number of identified individuals $(N)$ and Simpson's concentration index $(C)$ for chironomid midges emerging from plant and sediment.

\begin{tabular}{|c|c|c|c|c|c|c|c|c|c|c|c|c|c|c|c|c|c|c|}
\hline \multirow[b]{3}{*}{ Station } & \multicolumn{9}{|c|}{ Emergent plants } & \multicolumn{9}{|c|}{ Sediments } \\
\hline & \multicolumn{3}{|c|}{ May } & \multicolumn{3}{|c|}{ August } & \multicolumn{3}{|c|}{ November } & \multicolumn{3}{|c|}{ May } & \multicolumn{3}{|c|}{ August } & \multicolumn{3}{|c|}{ November } \\
\hline & $S$ & $N$ & $C$ & $S$ & $N$ & $C$ & $S$ & $N$ & $C$ & $S$ & $N$ & $C$ & $S$ & $N$ & $C$ & $S$ & $N$ & $C$ \\
\hline
\end{tabular}

Tsuchiurairi Bay

Median

$\begin{array}{rrrrrrrrrrrrrrrrrr}1 & 0 & 0 & & 3 & 17 & 0.7 & 3 & 10 & 0.4 & 0 & 0 & & 0 & 0 & & 0 & 0 \\ 2 & 3 & 13 & 0.4 & 1 & 1 & 1.0 & 1 & 3 & 1.0 & 3 & 5 & 0.4 & 1 & 1 & 1.0 & 0 & 0 \\ 3 & 4 & 15 & 0.4 & 4 & 11 & 0.3 & 2 & 11 & 0.5 & 2 & 5 & 0.7 & 0 & 0 & & 0 & 0 \\ 21 & 3 & 5 & 0.4 & 3 & 4 & 0.4 & 1 & 5 & 1.0 & 1 & 2 & 1.0 & 0 & 0 & & 0 & 0 \\ 22 & 10 & 196 & 0.4 & 3 & 15 & 0.4 & 2 & 11 & 0.7 & 6 & 9 & 0.2 & 1 & 31 & 1.0 & 0 & 0 \\ 23 & 3 & 176 & 0.8 & 3 & 4 & 0.4 & 1 & 5 & 1.0 & 7 & 28 & 0.4 & 0 & 0 & & 0 & 0 \\ & 3.8 & 67.5 & 0.5 & 2.8 & 8.7 & 0.5 & 1.6 & 7.5 & 0.8 & 3.2 & 8.2 & 0.5 & 0.3 & 5.3 & 1.0 & 0.0 & 0.0 \\ & 3 & 14 & 0.4 & 3 & 8 & 0.4 & 2 & 8 & 0.9 & 3 & 5 & 0.4 & 0 & 0 & 1.0 & 0 & 0\end{array}$

\section{Takahamairi Bay}

$\begin{array}{lrrrrrrrrrrrrrrrrrrr} & 13 & 3 & 36 & 0.6 & 4 & 49 & 0.9 & 0 & 0 & & 0 & 0 & & 0 & 0 & & 1 & 1 & 1.0 \\ & 14 & 4 & 10 & 0.4 & 6 & 42 & 0.4 & 7 & 39 & 0.5 & 1 & 1 & 1.0 & 4 & 80 & 0.9 & 2 & 2 & 0.5 \\ & 15 & 3 & 4 & 0.4 & 0 & 0 & & 3 & 43 & 0.6 & 2 & 2 & 0.5 & 2 & 17 & 0.9 & 1 & 4 & 1.0 \\ & 16 & 1 & 2 & 1.0 & 1 & 1 & 1.0 & 1 & 1 & 1.0 & 3 & 3 & 0.3 & 1 & 4 & 1.0 & 2 & 5 & 0.5 \\ & 17 & 2 & 7 & 0.6 & 5 & 14 & 0.3 & 3 & 12 & 0.4 & 2 & 2 & 0.5 & 3 & 51 & 0.9 & 0 & 0 & \\ & 18 & 0 & 0 & & 1 & 1 & 1.0 & 2 & 27 & 0.6 & 0 & 0 & & 4 & 17 & 0.5 & 1 & 5 & 1.0 \\ & 19 & 5 & 51 & 0.4 & 4 & 11 & 0.3 & 2 & 13 & 0.6 & 0 & 0 & & 0 & 0 & & 1 & 18 & 1.0 \\ \text { Mean } & 2.6 & 15.7 & 0.6 & 3.0 & 16.9 & 0.7 & 2.6 & 19.3 & 0.6 & 1.1 & 1.1 & 0.6 & 2.0 & 24.1 & 0.8 & 1.1 & 5.0 & 0.8 \\ \text { Median } & & 3 & 7 & 0.5 & 4 & 11 & 0.7 & 2 & 13 & 0.9 & 1 & 1 & 0.4 & 2 & 17 & 0.9 & 1 & 4 & 1.0\end{array}$

Edosakiiri Bay

$\begin{array}{lllllllllllllllllll}5 & 3 & 236 & 0.5 & 6 & 46 & 0.4 & 1 & 1 & 1.0 & 1 & 1 & 1.0 & 6 & 58 & 0.6 & 1 & 1 & 1.0\end{array}$

\begin{tabular}{lrrrrrrrrrrrrrrrrrrr} 
Main Basin & & & & & & & & & & & & & & & & & & \\
& 4 & 3 & 10 & 0.4 & 4 & 6 & 0.3 & 0 & 0 & & 1 & 3 & 1.0 & 1 & 5 & 1.0 & 0 & 0 & \\
& 6 & 3 & 19 & 0.5 & 0 & 0 & & 2 & 10 & 0.7 & 0 & 0 & & 0 & 0 & & 2 & 9 & 0.6 \\
& 7 & 3 & 63 & 0.7 & 3 & 5 & 0.4 & 1 & 1 & 1.0 & 2 & 2 & 0.5 & 0 & 0 & & 1 & 1 & 1.0 \\
& 8 & 3 & 33 & 0.7 & 2 & 10 & 0.5 & 2 & 12 & 0.9 & 3 & 10 & 0.5 & 0 & 0 & & 0 & 0 & \\
& 9 & - & - & - & - & - & - & - & - & - & 0 & 0 & & 5 & 9 & 0.2 & 1 & 1 & 1.0 \\
& 10 & 3 & 14 & 0.4 & 5 & 33 & 0.4 & 0 & 0 & & 1 & 1 & 1.0 & 2 & 2 & 0.5 & 1 & 4 & 1.0 \\
& 11 & 4 & 37 & 0.6 & 4 & 18 & 0.4 & 1 & 6 & 1.0 & 1 & 2 & 1.0 & 3 & 11 & 0.6 & 0 & 0 & \\
& 12 & 3 & 7 & 0.4 & 4 & 15 & 0.3 & 6 & 48 & 0.6 & 3 & 5 & 0.4 & 2 & 4 & 0.6 & 1 & 3 & 1.0 \\
& 20 & 4 & 8 & 0.3 & 4 & 10 & 0.3 & 3 & 16 & 0.6 & 1 & 1 & 1.0 & 0 & 0 & & 1 & 2 & 1.0 \\
Mean & 3.3 & 23.9 & 0.5 & 3.3 & 12.1 & 0.4 & 1.9 & 11.6 & 0.8 & 1.3 & 2.7 & 0.8 & 1.4 & 3.4 & 0.6 & 0.8 & 2.2 & 0.9 \\
Median & 3 & 17 & 0.5 & 4 & 10 & 0.4 & 2 & 8 & 0.8 & 1 & 2 & 1.0 & 1 & 2 & 0.6 & 1 & 1 & 1.0 \\
\hline Mean & 3.2 & 42.8 & 0.5 & 3.2 & 14.2 & 0.5 & 2.0 & 12.5 & 0.7 & 1.7 & 3.6 & 0.7 & 1.5 & 12.6 & 0.8 & 0.7 & 2.4 & 0.9 \\
Median & 3.0 & 13.5 & 0.4 & 3.5 & 10.5 & 0.4 & 2 & 10 & 0.7 & 1 & 2 & 0.5 & 1 & 2 & 0.9 & 1 & 1 & 1.0 \\
\hline
\end{tabular}

- No Data

hydrophytes in August and November. Cricotopus bicinctus also emerged mainly from hydrophytes. Cricotopus bimaculatus emerged exclusively from hydrophytes. Only one Cricotopus tricinctus emerged from each substratum.
Chironomid species associated with hydrophytes at Sta. 6, where cattails predominated in the hydrophyte community, did not differ from other stations with reed vegetation. D. peloch loris and $G$. tokunagai were abundant in May, while $H$. pilipes and L. tamakitanaides abounded 
Table 4. Numbers of individuals (converted in $\mathrm{m}^{-2}$ ) emerged from four types of sediments.

\begin{tabular}{|c|c|c|c|c|c|c|c|c|c|c|c|c|}
\hline \multirow[t]{2}{*}{ Species } & \multicolumn{4}{|c|}{ May } & \multicolumn{4}{|c|}{ August } & \multicolumn{4}{|c|}{ November } \\
\hline & $\begin{array}{c}\text { Sand } \\
(n=17)\end{array}$ & $\begin{array}{c}\text { Mud } \\
(n=2)\end{array}$ & $\begin{array}{l}\text { Plant } \\
\text { debris } \\
(n=3)\end{array}$ & $\begin{array}{c}\text { Cobble } \\
(n=1)\end{array}$ & $\begin{array}{c}\text { Sand } \\
(n=17)\end{array}$ & $\begin{array}{c}\text { Mud } \\
(n=2)\end{array}$ & $\begin{array}{c}\text { Plant } \\
\text { debris } \\
(n=3)\end{array}$ & $\begin{array}{l}\text { Cobble } \\
(n=1)\end{array}$ & $\begin{array}{c}\text { Sand } \\
(n=17)\end{array}$ & $\begin{array}{c}\text { Mud } \\
(n=2)\end{array}$ & $\begin{array}{l}\text { Plant } \\
\text { debris } \\
(n=3)\end{array}$ & $\begin{array}{c}\text { Cobble } \\
(n=1)\end{array}$ \\
\hline
\end{tabular}

Tanypodinae

Ablabesmyia monilis

Orthocladiinae

Cricotopus bicinctus

Cricotopus sylvestris

4

Cricotopus tricinctus

Hydrobaenus pilipes

150
10

Chironominae : Chironomini

Chironomus circumdatus

Chironomus kiiensis

Chironomus samoensis

Chironomus yoshimatsui

Cladopelma viridula

Cryptochironomus sp.

Dicrotendipes nervosus

Dicrotendipes pelochloris

Glyptotendipes tokunagai

Pentapedilum shirokanense

Polypedilum cultellatum

Polypedilum japonicum

Polypedilum nubeculosum

Polypedilum nubifer

$\begin{array}{rrr}4 & 4 \\ 4 & 102\end{array}$

4

2

5

34

6

34

6

Chironominae: Tanytarsini Cladotanytarsus vanderwulpi Tanytarsus oyamai Tanytarsus unagiseptimus

02

$2 \quad 8$ 
in November at Sta. 6.

Sediment types at sampling stations could be classified into four categories: sand, mud, plant debris and cobble (Fig. 1). Table 4 shows the list of chironomid species recovered from sediment in terms of the substratum type. Sediment was sandy at most (17) stations. Polypedilum nubifer emerged mainly from these sandy sediments. Although the remaining sediment types were low in number of replicates,

Table 5. A comparison of chironomid midges collected from Lake Kasumigaura in relation to substrates and methods of sampling

\begin{tabular}{|c|c|c|c|c|c|}
\hline \multirow[t]{2}{*}{ Species } & Pelagic & zone $^{a}$ & Littoral & zone $^{b}$ & Shoreside $^{c}$ \\
\hline & $\begin{array}{c}\text { Emergence } \\
\text { trap } \\
\text { sample }\end{array}$ & $\begin{array}{l}\text { Light } \\
\text { trap } \\
\text { sample }\end{array}$ & $\begin{array}{l}\text { Sediment } \\
\text { sample } \\
\text { (rearing) }\end{array}$ & $\begin{array}{c}\text { Plant } \\
\text { sample } \\
\text { (rearing) }\end{array}$ & $\begin{array}{c}\text { Net } \\
\text { sweeping } \\
\text { sample }\end{array}$ \\
\hline Clinotanypus sugiyamai TокUNAGA & $\mathrm{O}$ & 0 & & & \\
\hline Procladius culiciformis (Linnaeus) & $\mathrm{O}$ & $\mathrm{O}$ & & & \\
\hline Tokunagayusurika akamusi (TокUNAGA) & 0 & 0 & & & $\mathrm{O}$ \\
\hline Chironomus plumosus (LinNaeus) & 0 & 0 & & & 0 \\
\hline Harnischia complex sp. & 0 & & & & \\
\hline Glyptotendipes fujisecundus & 0 & & & 0 & \\
\hline Chironomus yoshimatsui & 0 & 0 & 0 & 0 & \\
\hline Dicrotendipes pelochloris & 0 & 0 & 0 & 0 & \\
\hline Cricotopus sylvestris & 0 & 0 & 0 & 0 & \\
\hline Polypedilum nubeculosum & 0 & & $\mathrm{O}$ & & \\
\hline Tanytarsini spp. & & 0 & & & \\
\hline Cricotopus spp. & & O & & & \\
\hline Chironomus nippodorsalis SASA & & 0 & & & \\
\hline Chironomus circumdatus & & 0 & 0 & & 0 \\
\hline Tanytarsus oyamai & & O & 0 & & 0 \\
\hline Chironomus kiiensis & & 0 & 0 & 0 & \\
\hline Glyptotendipes tokunagai & & 0 & 0 & 0 & \\
\hline Pentapedilum shirokanense & & 0 & 0 & 0 & \\
\hline Polypedilum nubifer & & 0 & 0 & 0 & \\
\hline Cricotopus tricinctus & & $\mathrm{O}$ & 0 & $\mathrm{O}$ & \\
\hline Cryptochironomus sp. & & & 0 & & \\
\hline Ablabesmyia monilis & & & 0 & & \\
\hline Chironomus samoensis & & & 0 & & \\
\hline Polypedilum japonicum & & & 0 & & \\
\hline Cricotopus bicinctus & & & O & 0 & \\
\hline Hydrobaenus pilipes & & & 0 & 0 & \\
\hline Cladopelma viridula & & & 0 & 0 & \\
\hline Dicrotendipes nervosus & & & 0 & 0 & \\
\hline Polypedilum cultellatum & & & 0 & 0 & \\
\hline Cladotanytarsus vanderwulpi & & & 0 & 0 & \\
\hline Tanytarsus unagiseptimus & & & 0 & 0 & \\
\hline Potthastia gaedii & & & & 0 & \\
\hline Cricotopus bimaculatus & & & & $\mathrm{O}$ & \\
\hline Limnophyes immucronatus & & & & 0 & \\
\hline Limnophyes tamakitanaides & & & & 0 & \\
\hline Orthocladius glabripennis & & & & 0 & \\
\hline Paratrichocladius rufiventris & & & & 0 & \\
\hline Stenochironomus membranifer & & & & 0 & \\
\hline Rheotanytarsus aestuarius & & & & 0 & \\
\hline
\end{tabular}

References

aWAKUMA (1992) ; ${ }^{b}$ Present study ; ${ }^{\circ}$ SASA (1979) 
i.e. one to three stations, several species seemed to be specific with specified substrates: $D i$ crotendipes pelochloris was associated with mud, Chironomus yoshimatsui with plant debris. Cricotopus sylvestris and Glyptotendipes tokunagai were primarily associated with cobbles, though they were rather more abundant on plant surfaces than in sediments. Tanytarsus oyamai emerged exclusively from sandy or muddy sediments rather than from attached material on hydrophytes.

\section{Discussion}

Species composition differed between hydrophyte and sediment at each station. Three species of Cricotopus, L. tamakitanaides, two species of Dicrotendipes and P. cultellatum were clearly abundant in hydrophyte samples, while Chironomus, Cryptochironomus, P. nubifer, and $T$. oyamai clearly abounded in sediments.

C. sylvestris uses emergent plants as the substrata for algal grazing (Menzie, 1981), or in some cases as food (Frost, 1924 cited in BERG, 1950 ; DARBy, 1962). A congener, Cricotopus trifasciatus, feeds on rice in Japan (NoDA et al., 1986), and there may be similar reasons why $C$. sylvestris was attracted to emergent plants in Lake Kasumigaura.

Iwakuma (1992) caught chironomid midges with emergence and light traps from May 1982 to December 1983 in Takahamairi Bay, the most eutrophic locale in Lake Kasumigaura. He recorded 17 chironomid species, 10 of which were also recorded in the present study. The chironomid fauna of Lake Kasumigaura is sum marized in Table 5. The number of species in the pelagic zone of Takahamairi Bay was smaller than that of the littoral hydrophyte zones. This difference between two zones is due to the variations in some environmental conditions. The most important difference between littoral and pelagic zones is the existence of hydrophyte stands in the littoral zone. Some authors reported the ecological significance of hydrophytes for chironomids, i.e. species composition of chironomids in vegetation differs from that in plain sediment; removal of aquatic weeds caused low diversity and high midge production (JOHNSON and MULLA, 1983); and the number of chironomid species correlated with the number of hydrophyte species among 14 ponds (Driver, 1977). Berg (1950) wrote that some species of Cricotopus, including C. sylvestris and Polypedilum, mine and feed on the tissues of hydrophytes, and some species of Glyptotendipes, Polypedilum, Microtendipes and Tanytarsus live in burrows beneath the epidermis of stems or leaves. We also observed $G$. tokunagai larvae beneath the epidermis of Typha stems or gathered on the cut ends of Phragmites in the laboratory. Emergent plants, therefore, might have supplied food for chironomid larvae in Lake Kasumigaura, though further studies are necessary to confirm the possibility of phytophagy on emergent plants by chironomid larvae in this lake.

As above, a higher diversity in chironomid fauna was observed in the hydrophyte zones than in the pelagic zones, although the pelagic chironomid fauna was not a subset of littoral chironomid fauna : the highly dominant species in the pelagic zone, Tokunagayusurika akamusi and Chironomus plumosus (Iwakuma, 1987, 1992), were not recovered from the littoral zone at all.

\section{Acknowledgements}

We thank Dr. Manabu SASA, President of Toyama University of International Studies, and Dr. Koichiro KawaI, Toyama Medical and Pharmaceutical University, for their helpful advice on the identification of chironomid midges. Thanks are also due to Mrs. H. SaIto for her laboratory work.

\section{摘要 \\ 霞ヶ浦抽水植物帯のユスリカ相}

霞ヶ浦水草帯のユスリカ相を明らかにするた め, 1986 年 5 月, 8 月, 11 月に各 1 回ヨシ植生内 より採取した水草及び底質から実験室内で羽化し たユスリカ成虫の種構成を調べた。 5 月と 8 月の 種構成はほ㴗同じで優占種は 10 種であった。主に 水草から羽化する種は 5 月に最も多く得られた。 これは水草表面の付着物中のクロロフィル $a$ 現 存量が 5 月に最も高く, 8 月に隇少したことと一 致した。また, 主に底質から羽化する種は 8 月に 
多かった。11月の優占種は 2 種で 5 月および 8 月 とは異なっていた。水草と水草周辺の底質とでは 種構成が異なっており，水草及び付着物から，種 数，個体数乞もに上り多くのコスりカ成虫が羽化 した。底質の種類とそのに生息するユスりカの種 類には関連がある可能性が示唆された。水草帯の 種構成はすでに報告されている沖带の種構成とは 異なっており，種数は水草帯の方が多かった。

\section{References}

Aizhki, M., T. Fukushima, S. Ebise, M. Hosomi, T. Iwakuma, T. Hanazato, N. Takamura, S. Nohara, A. Otsuki, T. Kawai, H. Shiraishi and Y. Nojiri (1988a): Comprehensive studies on effective use of natural ecosystems for water quality management. Environmental data for Lake Kasumigaura. I. Limnological data in Lake Kasumigaura. Res. Data Natl. Inst. Environ. Stud. 33: 1-66 (in Japanese).

Aizaki, M., S. Nohara, T. Kawai, T. Iwakuma, N. Takamura, T. Hanazato and A. Otsuki (1988b): Comprehensive studies on effective use of natural ecosystems for water quality management. Environmental data for Lake Kasumigaura. II. Limnological data in littoral zone of Edosakiiri Bay, Lake Kasumigaura. Res. Data Natl. Inst. Environ. Stud. 33: 67-105 (in Japanese).

Berg, C. O. (1950) : Biology of certain Chironomidae reared from Potamogeton. Ecol. Monogr. 20 : 85-101.

Boerger, H. J., H. F. Clifford and R. W. Davies (1982): Density and microdistribution of chironomid larvae in an Alberta brown-water stream. Can. J. Zool. 60: 913-920.

Cranston, P. S. and D. R. Oliver (1988): Addition and corrections to the Nearctic Orthocladiinae (Diptera: Chironomidae). Can. Ent. 120: 425 -462 .

DARBY, R. E. (1962) : Midges associated with California rice fields, with special reference to their ecology (Diptera, Chironomidae). Hilgardia 32 : 1-206.

Driver, E. A. (1977): Chironomid communities in small prairie ponds: some characteristics and controls. Freshwater Biology 7 : 121-133.

Frost, S. W. (1924): A study of the leaf-mining Diptera of North America. Mem. Cornell Univ. Agr. Exp. Sta. 78: 1-228.

Iwakuma, T. (1987) : Density, biomass and produc. tion of Chironomidae (Diptera) in Lake Kasumigaura during 1982-1986. Jpn. J. Limnol. 48 : $\mathrm{S} 59 \mathrm{-S} 75$.
Iwakuma, T. (1992): Emergence of Chironomidae from the shallow eutrophic Lake Kasumigaura, Japan. Hydrobiologia $245: 27-40$.

Iwakuma, T., M. Yasuno and Y. Sugaya (1984): Chironomid production in relation to phytoplankton primary production in Lake Kasumigaura, Japan. Verh. Internat. Verein. Limnol., 22 : 1150-1159.

Johnson, G. D. and M. S. Mulla (1983) : An aquatic macrophyte affecting nuisance chironomid midges in a warm-water lake. Environ. Entomol. $12: 266-269$.

Kondo, S. and S. Hamashima (1985): Chironomid midges emerged from aquatic macrophytes in reservoirs. Jpn. J. Limnol. $46: 50-55$.

Kondo, S. (1988) : Chironomids (Diptera) associated with reed, Phragmites communis Trin., in Nagoya City and its suburbs. Appl. Ent. Zool. $23: 353-354$.

Learner, M. A., P. R. Wiles and J. G. Pickering (1989): The influence of aquatic macrophyte identity on the composition of the chironomid fauna in a former gravel pit in Berkshire, England. Aquatics Insehts 11: 183-191.

Marker, A. F. H., C. A. Crowther and R. J. M. Gunn (1980): Methanol and acetone as solvents for estimating chlorophyll $a$ and phaeopigments by spectrophotometry. Arch. Hydrobiol. Beih. Ergebn. Limnol. 14: 52-69.

Mason, C. F. and R. J. Bryant (1975): Periphyton production and grazing by chironomids in Alderfen Broad, Norfolk. Freshwater Biol. 5 : 271-277.

McGgaha, Y. J. (1952): The limnological relations of insects to certain aquatic flowering plants. Trans. Am. Micros. Soc. 71: 355-381.

Menzie, C. A. (1981): Production ecology of Cricotopus sylvestris (FABricius) (Diptera: Chironomidae) in a shallow estuarine cove. Limnol. Oceanogr. 26 : 467-481.

Miyadi, D. (1932): Studies on the bottom fauna of Japanese lakes. III. Lakes of the Kwanto plain. Jpn. J. Zool. 3: 1-18.

Noda, H., M. Miyazaki and H. Hashimoto (1986): Injury to rice leaves by chironomid larvae (Diptera: Chironomidae). Jpn. J. Appl. Ent. Zool. 30: 66-68 (in Japanese).

Pinder, L. C. V. (1978): A key to the adult males of the British Chironomidae (Diptera). Freshwater Biological Assoc. Publ. No. 37. 1: 1 -169 ; 2 : Fig. 77-184.

SaKural, Y. (1981): Changes of flora, vegetation area and biomass of aquatic plants in the 
recent progress of eutrophication in Lake Kasumigaura. Res. Rep. Natl. Inst. Environ. Stud. 22 : 229-279 (in Japanese).

SASA, M. (1978): A comparative study of adults and immature stages of nine Japanese species of the genus Chironomus (Diptera, Chironomidae). Res. Rep. Natl. Inst. Environ. Stud. 3 : 63pp.

SASA, M. (1979): A morphological study of adults and immature stages of 20 Japanese species of the family Chironomidae (Diptera). Res. Rep. Natl. Inst. Environ. Stud. 7 : 148pp.

SASA, M. (1985a): Studies on the chironomids collected from lakes in Southern Kyushu (Diptera, Chironomidae). Res. Rep. Natl. Inst. Environ. Stud. 83 : 25-99.

SASA, M. (1985b): Studies on the chironomids collected from lakes in the Mount Fuji Area (Diptera, Chironomidae). Res. Rep. Natl. Inst. Environ. Stud. $83: 101-154$.

SASA, M. (1989) : Chironomidae of Japan : checklist of species recorded, key to males and taxonomic notes. Res. Rep. Natl. Inst. Environ. Stud. $125: 177 \mathrm{pp}$.

Sasa, M. and H. Hasegawa (1983): Chironomid midges of the tribe Chironomini collected from sewage ditches, eutrophicated ponds, and some clean streams in the Ryukyu Islands, southern Japan. Jpn. J. Sanit. Zool. 34 : 305-341.

SaSA, M. and K. KawaI (1987): Studies on the chironomid midges of Lake Biwa (Diptera, Chironomidae). L. Biwa Study Monogr. 3 : 119pp. Simpson, E. H. (1949): Measurement of diversity.
Nature $163: 688$.

Smock, L. A. and D. L. Stoneburner (1980): The response of macroinvertebrates to aquatic macrophyte decomposition. Oikos $35: 397$ -403 .

Takamura, N., T. Iwakuma, M. Aizaki and M. Yasuno (1990): Primary production of epiphytic algae and phytoplankton in the littoral zone of Lake Kasumigaura. Marine Microbial Food Webs 4: 239-255.

Tsuda, M. and I. Morishita (1973): Zoobenthos of sublittoral zone, p. 56-62. In : Lake Kasumigaura Work Office of Ministry of Construction (ed.), Report of Biological Survey in Lake Kasumigaura. Lake Kasumigaura Work Office of Ministry of Construction and Water Resources Development Corporation (in Japanese).

WeTzEL, R. G. and H. Corners (1979): The role of the littoral zone and detritus in lake metabolism. Arch. Hydrobiol. Beih. Ergebn. Limnol. 13: 145-161.

（著者：上野隆平・岩熊敏夫・野原精一，国立環境 研究所生物圈環境部，テ305 つくば市小野川 16-2； Ryuhei Ueno, Toshio Iwakuma and Seiichi Nohara, Environmental Biology Division, National Institute for Environmental Studies, Onogawa, Tsukuba, Ibaraki 305)

Received : 22 June 1993

Accepted : 23 August 1993 